\title{
Kinetics of optically generated defects in hydrogenated amorphous silicon
}

\author{
Y. F. Chen, S. F. Huang, and W. S. Chen \\ Department of Physics, National Taiwan University, Taipei, Taiwan, Republic of China
}

(Received 16 April 1991; revised manuscript received 29 July 1991)

\begin{abstract}
The time dependence of optically induced degradation in hydrogenated amorphous silicon $(a-\mathrm{Si}: \mathrm{H})$ has been measured at various temperatures. It is found that the degradation follows the stretchedexponential law with the stretching parameter $\beta$ and the relaxation time constant $\tau$ independent of the sample temperature. This result is explained by the fact that the defects in an amorphous solid are distributed exponentially in energy and the absorbed photon transfers its energy into a local mode of the defect to raise the local temperature to a value of $T_{x}=408 \mathrm{~K}$. We conclude that the sample temperature will not affect the process of defect conversion if it is smaller than $T_{x}$. We further predict that if it is larger than $T_{x}$, the process will show the temperature dependence. The prediction is consistent with the previous measurements. Thus, our results may serve as a solution to the hitherto unresolved issue of the temperature dependence of the creation of dangling bonds and establish its underlying mechanism. The observed independence of the relaxation parameters $\beta$ and $\tau$ on the sample temperature does not favor the hydrogen-diffusion weak-bond model for the optically induced degradation in $a-\mathrm{Si}: \mathrm{H}$, in which the hydrogen atom moves a microscopic distance to break a weak bond. We also show that the $t^{1 / 3}$ time dependence can only describe a certain range of our measurements.
\end{abstract}

\section{INTRODUCTION}

Since Staebler and Wronski ${ }^{1}$ discovered the optically induced degradation in hydrogenated amorphous silicon ( $a-\mathrm{Si}: \mathrm{H})$, many efforts have been devoted to understanding its underlying mechanism. Even though several models have been presented, the problem is still unresolved. An appropriate model plays an important role in understanding, and hopefully controlling, this effect. The time and temperature dependence of this effect are the significant measurable quantities that can provide insights into the mechanism involved. However, these measurements have been examined rather incompletely. In this paper we report data to fill this gap in knowledge. We have observed that the time dependence of the defects generated by illumination throughout the entire measurement follows the stretched-exponential law given by

$$
N=N_{s}-\left(N_{s}-N_{0}\right) \exp \left[-(t / \tau)^{\beta}\right],
$$

where $N_{s}$ is the saturated value of the defect density, $N_{0}$ is the initial value of the defect density before optical degradation, $\beta$ is the stretching parameter, and $\tau$ is the defect-generation time constant. The striking result is that both the stretching parameter and the generation time constant do not depend on the sample temperature. We have also found that the power-law dependence $N \propto t^{1 / 3}$ can only be used to describe a certain portion of the measurement. The implication of our results for some of the existing models will be discussed. A theory to explain our experimental results will be developed. Our results will be used to resolve the discrepancy between the temperature dependence of the defect creation and existing models.

\section{EXPERIMENTAL DETAILS}

Undoped $a$-Si:H samples were prepared by using a $\mathrm{rf}$ glow-discharge decomposition of pure silane. The deposition conditions were as follows: substrate temperature, $250^{\circ} \mathrm{C}$; chamber pressure, 1 mbar; rf power density, 0.1 $\mathrm{W} / \mathrm{cm}^{2}$; rf frequency, $13.56 \mathrm{MHz}$. The samples have been characterized by various measurements. ${ }^{2}$ The properties of the samples are as follows: initial dark conductivity, $10^{-9}(\Omega \mathrm{cm})^{-1} ; 100 \mathrm{~mW} / \mathrm{cm}^{2}$ photoconductivity, $5 \times 10^{-4}(\Omega \mathrm{cm})^{-1}$; optical band gap, $1.75 \mathrm{eV}$; activation energy, $0.75 \mathrm{eV}$, hydrogen content, 5-10 at. \%; sample thickness, $2 \mu \mathrm{m}$. Optical exposures were carried out in vacuum with light of $50 \mathrm{~mW} / \mathrm{cm}^{2}$ intensity from a $\mathrm{He}-\mathrm{Ne}$ laser focused onto the film. Before the optical exposure, all samples were annealed at about $150^{\circ} \mathrm{C}$ for $1 \mathrm{~h}$ in vacuum. Conductivity measurements were performed for samples deposited on a Corning 7095 glass substrate with a conventional coplanar configuration using a HewlettPackard 4140 B picoammeter.

\section{THEORY}

The configurational ground state of an amorphous solid is not given by an absolute and isolated minimum of the total potential energy, but rather by a flexible arrangement of the atoms allowing configurations with different local minima of the potential energy separated by a continuous distribution of potential barriers. ${ }^{3}$ The possibility of transitions between adjacent local potential minima can thus lead to a number of peculiar phenomena which include the optically induced degradation in $a$ $\mathrm{Si}: \mathrm{H}$. The transitions are usually represented by a twolevel system as shown in Fig. 1. In equilibrium, most of the defects are in state $A$. If an external excitation is ap- 


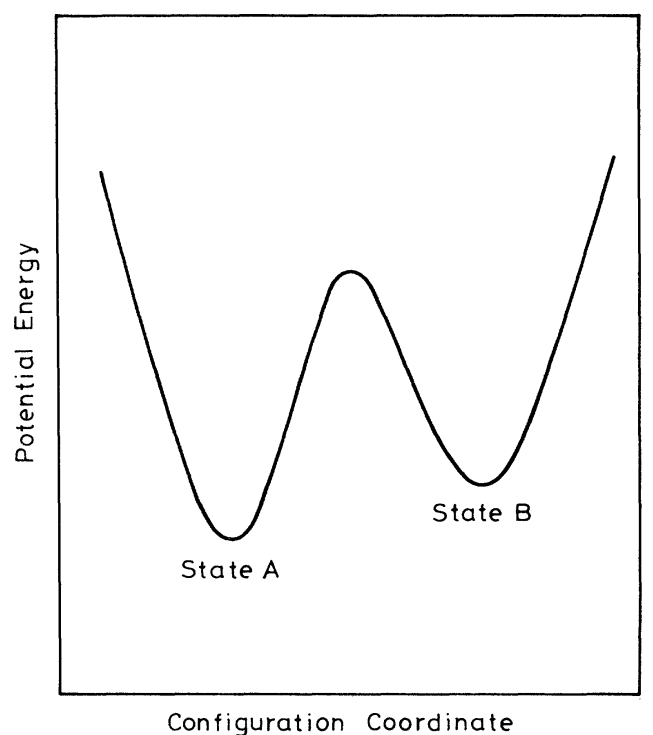

FIG. 1. The two-level configuration-coordinate diagram of a metastable defect.

plied, transition from state $A$ to $B$ becomes possible. As a consequence, the occupancy of state $A$ will be gradually reduced with the time of excitation. In the following we will present the mathematical formalism for the time dependence of the relaxation of state $A$ to $B$. The derivation is similar to that given by several previous authors. ${ }^{4-7}$ Here, we only briefly outline the procedures.

According to the thermodynamic equilibrium arguments, ${ }^{8}$ all defects in an amorphous solid are distributed exponentially in energy characterized by the temperature $T_{0}$. It can be written as

$$
n_{A}(E)=\frac{N_{A}}{k T_{0}} e^{\left(E-E_{m}\right) / k T_{0}},
$$

where $n_{A}(E)$ is the density of defects in state $A$ per unit energy, $N_{A}$ is the total population in state $A$, and $E_{m}$ is the maximum barrier height for the transition from state $A$ to $B$. The rate of change of $n_{A}(E)$ is given by

$$
\frac{d n_{A}(E)}{d t}=-n_{A}(E) R_{A-B}(E),
$$

where $R_{A-B}(E)$ is the transition probability per unit time per defect from state $A$ to $B$. For a thermally activated process,

$$
R_{A-B}(E)=R_{0} e^{-E / k T},
$$

where $R_{0}$ is the transition rate constant. Using the assumed distribution functions, the rate of change of the total population in state $A$ is

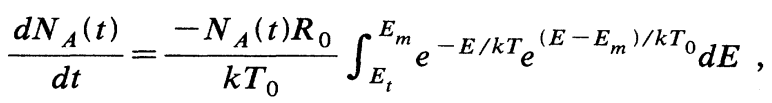

where $E_{t}$ is the lower limit of the integration which separates those states that reconfigure during the mea- surement from those that remain in equilibrium. ${ }^{7} E_{t}$ is given by

$$
E_{t}=k T \ln \left(R_{0} t\right) .
$$

Thus,

$$
\frac{d N_{A}(t)}{d t}=-N_{A}(t) \frac{\left(R_{0} t\right)^{\beta-1}}{\beta-1},
$$

where $\beta=T / T_{0}$. To obtain Eq. (7), we have assumed that the lower limit dominates the integral since the argument of the integral decreases exponentially at high energy. From Eq. (7), we can see that the change of defects following a power-law time dependence is simply the consequence of the defects distributed exponentially in energy. Thus, the time dependence of the relaxation of state $A$ to $B$ is

$$
N_{A}(t)=N_{A}(0) e^{-(t / \tau)^{\beta}}
$$

where

$$
\tau=R_{0}{ }^{-1} e^{E_{\tau} / k T}
$$

with

$$
E_{\tau}=E_{m}+k T_{0} \ln (1-\beta),
$$

where $\tau$ is the time constant for the defects in state $A$ to relax to $B$, and $E_{\tau}$ is the activation energy for this relaxation. Equation (8) demonstrates that the relaxation of state $A$ follows a stretched-exponential function rather than a simple exponential function. In the studies of the optically induced degradation in $a-\mathrm{Si}: \mathrm{H}$, we usually measure the physical quantities related to the defects in state $B$ rather than in state $A$. This is due to the fact that state $B$ contains a neutral dangling bond which can easily be detected by electron-spin-resonance or photoconductivity measurements. To convert Eq. (8) into the time dependence of the total population in state $B$, we use the following relations:

$$
N_{A}(0)=N_{B}(\infty)-N_{B}(0)
$$

and

$$
N_{A}(t)=N_{B}(\infty)-N_{B}(t),
$$

where $N_{A}(0)$ is the initial population of defects in state $A, N_{B}(\infty)$ and $N_{B}(0)$ are the saturated and initial population of defects in state $B$, respectively, and $N_{A}(t)$ and $N_{B}(t)$ are the population of defects in state $A$ and $B$ after the exposure time $t$, respectively. Thus, the time dependence of defects in state $B$ is

$N_{B}(t)-N_{B}(0)=\left[N_{B}(\infty)-N_{B}(0)\right]\left(1-e^{-(t / \tau)^{\beta}}\right)$.

Equation (13) can be rewritten as

$$
\ln f=\beta \ln t-\beta \ln \tau,
$$

where

$$
f=\ln \left\{\left[N_{B}(\infty)-N_{B}(0)\right] /\left[N_{B}(\infty)-N_{B}(t)\right]\right\} .
$$

A plot of $\ln f$ versus $\ln t$ yields a straight line with a slope $\beta$ and an intercept $\beta \ln \tau$. It is worth emphasizing that the 
main determinant of the above derivation is the underlying disorder in the amorphous solids. As long as all defects in amorphous solids are distributed exponentially in energy, the relaxation of any thermally activated processes involving the defects will follow the stretchedexponential function, which does not depend on the underlying mechanisms.

If the energy for defect conversion is supplied by the absorbed photon rather than by the thermal energy, the defect relaxation is a nonthermal process and independent of the temperature. This process can be understood by assuming that the absorbed photon transfers its energy into a local mode of the defect to raise the local temperature to a value of $T_{x}{ }^{7,9}$ The kinetics of this process can be obtained by replacing $T$ by $T_{x}$ in Eq. (13). The relaxation of defects still follows the stretched-exponential function. The parameters in the function, however, are independent of temperature; for instance, the stretching parameter

$$
\beta=T_{x} / T_{0}
$$

and the relaxation time constant

$$
\tau=R_{0}{ }^{-1} e^{E_{\tau} / k T_{x}} \text {. }
$$

We may assume that the energy transformation from the incident photon to the defect is due to the recombination of the photon-excited carriers. After the carriers are created, they may be trapped by the defects and recombine through the defects. The energy released by the recombination is located locally at the position of the defect and can be easily absorbed and raise the local temperature of the defect. Thus, even increasing the illumination intensity can enhance the rate of defect relaxation; it does not affect the local temperature $T_{x}$ since $T_{x}$ is determined by the releasing energy per recombination.

\section{EXPERIMENTAL RESULTS AND DISCUSSION}

In Fig. 2 we show a typical example of the degradation of the photocurrent as a function of exposure time. To use Eq. (13) to describe our data, we assume that the photoconductivity is inversely proportional to the number of dangling bonds. This assumption has been predicted theoretically ${ }^{3,10}$ and verified experimentally. ${ }^{3,11}$ According to these calculations, the photocurrent is linearly proportional to the light intensity, i.e., we have $\sigma_{\mathrm{ph}} \propto I^{r}$, where $r=1$. Figure 3 gives the lux-ampere characteristics investigated at $T=303 \mathrm{~K}$. Clearly, the value of the parameter in question is $r \approx 1$. This demonstrates the feasibility of using the above assumption.

In Fig. 4 we show the fitting of our data to Eq. (14). We can clearly see that a plot of $\ln f$ versus $\ln t$ yields a straight line throughout the entire measurement. A striking result is found that the stretching parameter $\beta$ obtained from the slope and the relaxation time constant $\tau$ from the intercept are independent of the sample temperature. The values of $\beta$ and $\tau$ are $0.68 \pm 0.02$ and $(9.0 \pm 0.1) \times 10^{3} \mathrm{sec}$, respectively. In an alternative approach, we use the same values of $\beta$ and $\tau$ to fit our data to Eq. (13) without taking the logarithm as shown in Fig.

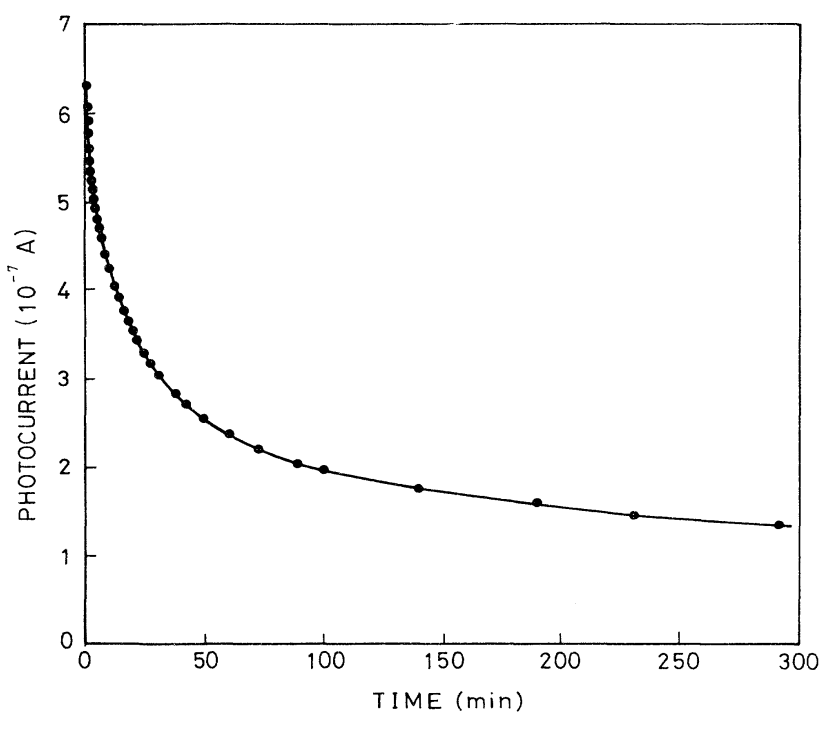

FIG. 2. Degradation of the photocurrent as a function of illumination time at $T=294 \mathrm{~K}$.

5. The good agreement between the theory and experiment thus demonstrates that the optically induced degradation in $a-\mathrm{Si}: \mathrm{H}$ follows the stretched-exponential relaxation.

If we take the characteristic temperature $T_{0}=600 \mathrm{~K}$ in $a-\mathrm{Si}: \mathrm{H},{ }^{5}$ the corresponding local temperature $T_{x}$ of defects due to the absorbed photon is about $408 \mathrm{~K}$. Since all our measurements were performed in the temperature range lower than this temperature, we thus expect that the results should not depend on the sample temperature, which is just what we have observed. We further predict that if the sample temperature is higher than $T_{x}$, the en-

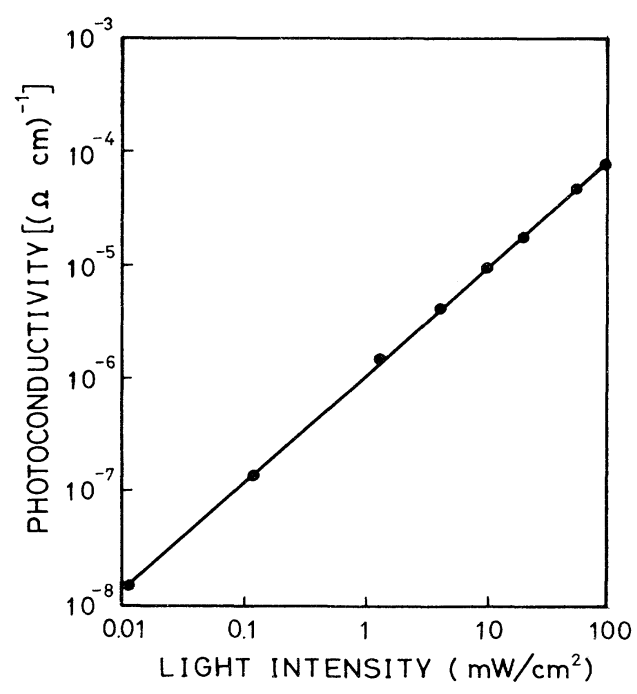

FIG. 3. Intensity dependence of the steady-state photoconductivity at $T=303 \mathrm{~K}$. 


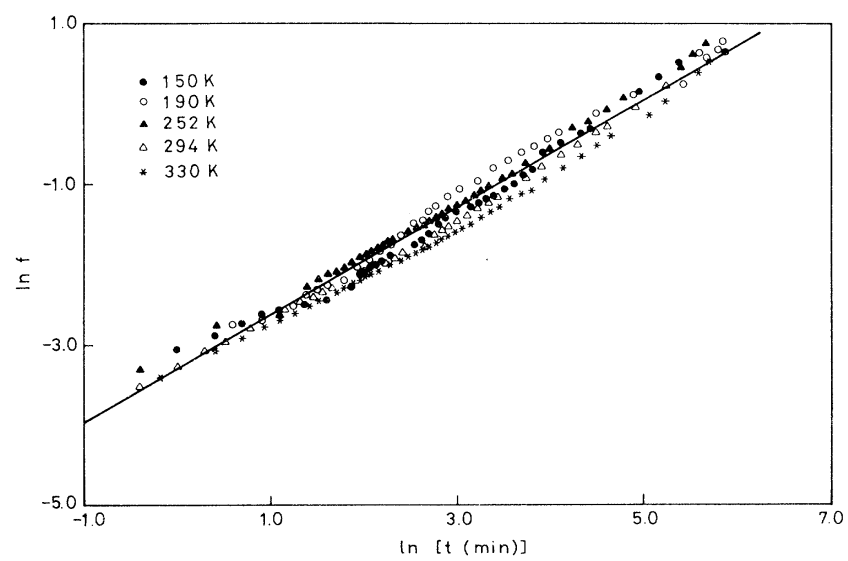

FIG. 4. Double-logarithmic plot of $f=\ln \left\{\left[N_{B}(\infty)\right.\right.$ $\left.\left.-N_{B}(0) / N_{B}(\infty)-N_{B}(t)\right]\right\}$ vs exposure time for a variety of sample temperatures. The solid line shows that the time dependence of the defect conversion at all temperatures follows a single stretched-exponential function. The units for all plots are in minutes.

ergy supplied by the thermal energy of the sample can have a dominant contribution to the defect conversion, and hence the process will depend on the sample temperature. Indeed, Crandall ${ }^{12}$ has measured the optically induced degradation in $a-\mathrm{Si}: \mathrm{H}$ at temperature higher than $425 \mathrm{~K}$. He found that the activation energy for this process is as large as $1 \mathrm{eV}$, which is completely different from that of the measurements around room temperature. In

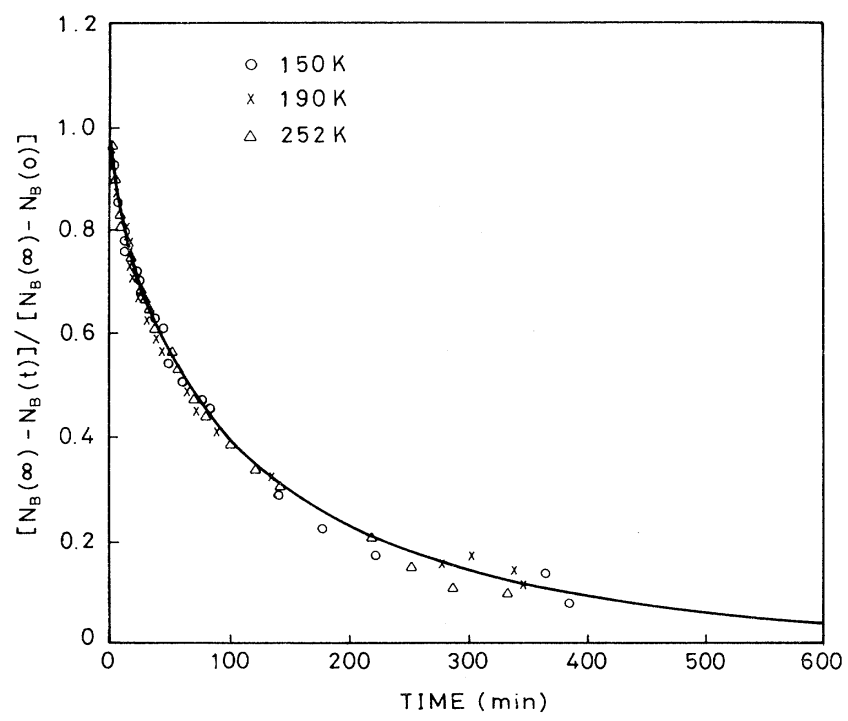

FIG. 5. Plots of $\left[N_{B}(\infty)-N_{B}(t)\right] /\left[N_{B}(\infty)-N_{B}(0)\right]$ vs exposure time for a variety of sample temperatures. The solid line shows that the time dependence of the defect conversion at all temperatures follows a single stretched-exponential function. The units for all plots are in minutes. the region around room temperature, the dependence of the optically induced degradation on temperature is rather weak. ${ }^{3}$ The discrepancy between the temperature dependence of the creation of dangling bonds and existing models has remained an unresolved issue for a long time. Our results may serve as a solution to this problem and may establish the underlying mechanism. Previous$1 y$, the stretched-exponential function has been used to describe optically induced defects in $a-\mathrm{Si}: \mathrm{H}$ by Redfield and Bube. ${ }^{13}$ However, as they pointed out, ${ }^{14}$ the temperature dependence of the kinetics has not been verified, and neither has the physics underlying their rate equation been specified.

Our discussion here is based on the assumption that the optically induced degradation in $a-\mathrm{Si}: \mathrm{H}$ is a process of thermal activation over barriers. According to our measurements below the local temperature $T_{x}$, one may question the above assumption. Since the process does not depend on the sample temperature at this temperature region, it is possible that defects are created by a mechanism other than thermal activation over barriers. However, this assumption is justified according to the measurements by Crandall ${ }^{10}$ as described above. The defect creation is a thermally activated process above the local temperature $T_{x}$.

An alternative degradation kinetics which has been widely used is proposed by Stutzmann et al. ${ }^{3}$ They assumed that the change of defects in state $B$ is due to the annihilation of electrons and holes, and the rate equation is given by

$$
\frac{d n_{B}(t)}{d t} \propto n p,
$$

where $n$ and $p$ are the densities of electrons and holes, respectively. An increase of the defects in state $B$ will reduce the lifetimes of electrons and holes, and therefore their densities. Assuming $n$ and $p$ are inversely proportional to $N_{B}(t)$, the rate equation at long-time limit becomes

$$
\frac{d N_{B}(t)}{d t} \propto \frac{1}{N_{B}^{2}(t)} .
$$

This leads to the time dependence of $N_{B}(t)$ following $t^{1 / 3}$. As shown in Fig. 6, this power-law time dependence can only be fitted to a certain range of our measurement. Thus, we may consider that this dependence is an approximation of the stretched-exponential relaxation. Note that the derivation at short times is compatible with predictions of the Stutzmann model, ${ }^{3}$ which cannot be ruled out.

We now compare the results of our measurements and the main microscopic models for the optically induced degradation in $a-\mathrm{Si}: \mathrm{H}$. Two main microscopic models have been proposed to explain this effect. ${ }^{7}$ These are the weak-bond-breaking and charge-trapping-defect models. For the weak-bond-breaking model, many authors ${ }^{15-17}$ proposed that dangling bonds are created when a weak $\mathrm{Si}-\mathrm{Si}$ bond is broken following the absorption of a photon. By comparing the energies of the weak-bond state after the excitation of an electron from a bonding orbital 


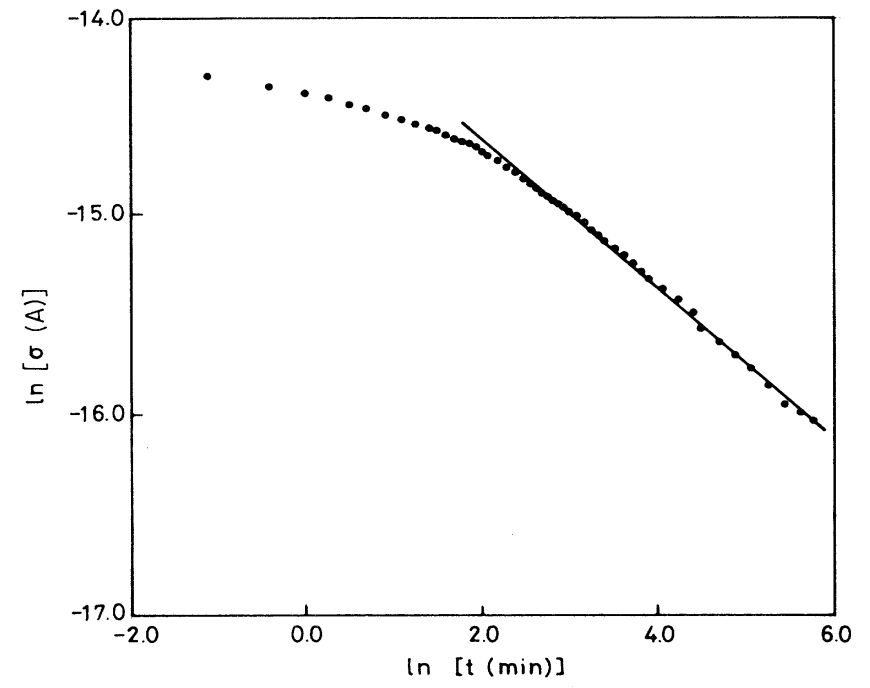

FIG. 6. Double-logarithmic plot of photoconductivity vs exposure time for the sample at $200 \mathrm{~K}$. The solid line shows that the photoconductivity follows a $t^{1 / 3}$ time dependence. The unit for the plot is in minutes.

to an antibonding orbital and the final state containing two dangling bonds, Stutzmann ${ }^{18,19}$ showed that the weak bond should break. To stabilize the two dangling bonds, several suggestions have been proposed. One of the possibilities is that without involving a hydrogen atom, ${ }^{18,19}$ local atomic rearrangement between the weak bond and two dangling bonds can form a potential barrier to prevent the defect conversion. However, many others $^{5,20,21}$ proposed that a $\mathrm{Si}-\mathbf{H}$ bond is broken after the absorption of photons and the hydrogen atom diffused to a weak-bond site and to create dangling bonds. For the charged-trapping-defect model, Adler ${ }^{22}$ proposed that during illumination with band-gap light, electrons and holes are trapped on the positive and negative dangling-bond defects. The resulting neutral dangling bonds will relax to become stable defects. Since $\mathrm{Si}-\mathrm{Si}$ bond angles have to change during the relaxation process, there is a barrier to overcome.

Since all defects in an amorphous solid are distributed exponentially in energy, ${ }^{8}$ the relaxation of any thermally activated processes involving the defects will follow the stretched-exponential function according to our discussion in Sec. III. Thus, the stretched-exponential relaxation as observed cannot be used to discriminate any microscopic models discussed above. However, the hydrogen-diffusion weak-bond model proposed by Jackson ${ }^{6,18}$ is inconsistent with the fact that the stretching pa- rameter $\beta$ and the relaxation time constant $\tau$ do not depend on the sample temperature. In this model, after breaking away from a $\mathrm{Si}-\mathrm{H}$ bond, the hydrogen atom must move a macroscopic distance to break a weak bond. This movement is a thermally activated process which will lead to the relaxation parameters $\beta$ and $\tau$ depending on the sample temperature. Thus, our measurements do not favor the hydrogen-diffusion weak-bond model proposed by Jackson. ${ }^{6,8}$ However, our results cannot be used to rule out all the models involving a hydrogen atom to explain the optically induced degradation in $a-\mathrm{Si}: \mathrm{H}$. For example, in the Stutzmann model ${ }^{3}$ the hydrogen is assumed to switch only to a nearest-neighbor site. The conclusion here is consistent with the experimental result for the role of hydrogen in the optically induced degradation by Stutzmann et al. ${ }^{23}$ They showed that if hydrogen were involved in the weak-bond breaking, it could not be the rate-limiting step.

\section{SUMMARY}

We have shown that the time dependence of the optically induced degradation in $a-\mathrm{Si}: \mathrm{H}$ at various temperatures follows a single stretched-exponential function with the stretching parameter $\beta$ and the relaxation time constant $\tau$ being independent of the sample temperature. These results can be explained by the fact that the defects in an amorphous solid are distributed exponentially in energy and the absorbed photon transfers its energy into a local mode of the defect to raising the local temperature to a value of $T_{x}$. The value of $T_{x}$ obtained is about 408 $K$. We conclude that the sample temperature will not affect the process of defect conversion if it is lower than $T_{x}$. However, if it is higher than $T_{x}$, the process of defect conversion will show the temperature dependence. This conclusion can be used to resolve the discrepancy between the temperature dependence of the creation of dangling bonds and existing models. The observed independence of the relaxation parameters $\beta$ and $\tau$ on the sample temperature does not favor the hydrogendiffusion weak-bond model for the optically induced degradation in $a-\mathrm{Si}: \mathrm{H}$ as proposed by Jackson, ${ }^{6,18}$ in which the hydrogen atom moves a microscopic distance to break a weak bond. We have also shown that the $t^{1 / 3}$ time dependence can only describe a certain range of our measurements. Thus, this power-law time dependence is an approximation of the stretched-exponential relaxation.

\section{ACKNOWLEDGMENTS}

We thank Dr. W. J. Tzeng for providing the measurement of lux-ampere characteristics. This work was supported in part by the National Science Council of the Republic of China.
${ }^{1}$ D. L. Staebler and C. R. Wronski, Appl. Phys. Lett. 31, 292 (1977).

${ }^{2}$ W. S. Chen, M. Sc. thesis, National Taiwan University, Taipe, Taiwan, 1990.
${ }^{3}$ M. Stutzmann, W. B. Jacksonn, and C. C. Tsai, Phys. Rev. B 32, 23 (1985).

${ }^{4}$ M. Campos, J. A. Giacometti, and M. Silver, Appl. Phys. Lett. 34, 226 (1979). 
${ }^{5}$ J. Kakalios, R. A. Street, and W. B. Jackson, Phys. Rev. Lett. 59, 1037 (1987).

${ }^{6}$ W. B. Jackson, Phys. Rev. B 38, 3595 (1988).

${ }^{7}$ R. S. Crandall, Phys. Rev. B 43, 4057 (1991).

${ }^{8}$ Y. Bar-Yam, D. Adler, and J. D. Joannopoulos, Phys. Rev. Lett. 57, 467 (1988).

${ }^{9}$ W. B. Jackson, Philos. Mag. Lett. 59, 103 (1989).

${ }^{10}$ L. A. Balagurov, Ya. Ya. Kyutte, E. M. Omel'yanovskii, S. A. Ostashko, L. E. Stys, and M. G. Foigel', Fiz. Tekh. Poluprovodn. 19, 1046 (1985) [Sov. Phys. Semicond. 19, 641 (1985)].

${ }^{11}$ I. P. Zvyagin, I. A. Kurova, N. V. Meleshko, and N. N. Ormont, Fiz Tekh. Poluprovodn. 24, 1726 (1990). [Sov. Phys. Semicond. 24, 1078 (1990)].

${ }^{12}$ R. S. Crandall, Phys. Rev. B 36, 2645 (1987).

${ }^{13}$ D. Redfield and R. H. Bube, Appl. Phys. Lett. 54, 1037 (1989).
${ }^{14}$ R. H. Bube, L. Echeverria, and D. Redfield, Appl. Phys. Lett. 57, 79 (1990).

15J. Pankove and J. E. Berkeyheiser, Appl. Phys. Lett. 37, 705 (1980).

16S. R. Elliott, Philos. Mag. B 39, 349 (1979).

${ }^{17}$ D. L. Staebler and C. R. Wronski, J. Appl. Phys. 51, 3262 (1980).

${ }^{18}$ M. Stutzmann, Philos. Mag. B 56, 63 (1987).

${ }^{19}$ M. Stutzmann, Philos. Mag. B 60, 531 (1989).

${ }^{20}$ W. B. Jackson, Phys. Rev. B 41, 10257 (1990).

${ }^{21}$ Y. F. Chen and C. K. Wong, Phys. Status Solidi B 157, 101 (1990).

${ }^{22}$ D. Adler, Solar Cells 9, 133 (1982).

${ }^{23}$ M. Stutzmann, W. B. Jackson, A. J. Smith, and R. Thompson, Appl. Phys. Lett. 48, 62 (1985). 